\title{
New DAQ for the HJET polarimeter at RHIC
}

\author{
A. A. Poblaguev* for the RHIC/CNI polarimetry group ${ }^{\dagger}$ \\ Brookhaven National Laboratory, Upton, NY 11973, USA \\ E-mail: poblaguevabnl.gov
}

In RHIC Run 15 a new DAQ for the polarized hydrogen jet target polarimeter (HJET) was tested and employed. The DAQ based on Jefferson Lab 12 bit $250 \mathrm{MHz}$ flash ADC and new set of silicon detectors allowed us to improve quality of the data analysis. Comparison of different methods of energy calibration of the detectors will be reported. Analysis of the signal waveform shape allowed us to separate stopped and punched through recoil protons. Such a separation results in an extension of the energy range of detected protons as well as in a suppression of inelastic background. Systematic errors of the beam polarization measurements are discussed. Preliminary results for the $100 \mathrm{GeV}$ polarized protons analyzing power on $\mathrm{Au}$ and $\mathrm{Al}$ are presented.

XVIth International Workshop in Polarized Sources, Targets, and Polarimetry, PSTP2015,

14-18 September 2015,

Bochum, Germany

* Speaker.

${ }^{\dagger}$ I. Alekseev, E. Aschenauer, G. Atoian, K.O. Eyser, H. Huang, D. Kalinkin, Y. Makdisi, A. Poblaguev, W. Schmidke, D. Svirida, G. Webb, A. Zelenski. 


\section{Introduction}

Monitoring of the proton beam polarization is an important part of operation of the RHIC polarized proton complex [1]. The polarized hydrogen jet target polarimeter (HJET) [2, 3] is employed to measure absolute polarization of the both RHIC proton beams, blue and yellow. Schematically the HJET consist of the polarized jet target and silicon strip detectors as sketched in Fig. 1.

Since blue and yellow beams are vertically separated at the HJET location, polarization of both beams are measured concurrently by studying left/right asymmetry of recoil proton production in elastic $p p$ scattering.

To isolate recoil protons, the detected signal time $t$ is compared with measured amplitude $A$

$$
T=\frac{m_{p} L^{2}}{2\left(t-t_{0}\right)^{2}}=g A+E_{\mathrm{loss}}\left(g A, x_{\mathrm{DL}}\right)
$$

where $T$ is proton kinetic energy, $m_{p}$ is proton mass, $L=77 \mathrm{~cm}$ is the distance to the detectors, $t_{0}$ is time offset, $g$ is the ADC gain, $x_{\mathrm{DL}} \approx 0.37 \mathrm{mg} / \mathrm{cm}^{2}$ is the entrance window (dead-layer) thickness, and $E_{\text {loss }}\left(A, x_{\mathrm{DL}}\right)$ is the energy loss in dead-layer calculated using the stopping power tables[5]. For each Si strip, the values of $t_{0}, g$, and $x_{\mathrm{DL}}$ are found in detector calibration. The event selection cuts corresponding to Eq. 1.1 are shown by black lines in Fig. 2. The width of this cut is defined by the beam bunch length.

For elastic $p p$ scattering there is a strict dependence between recoil proton kinetic energy $T$ and $z$-coordinate in the detector (or the scattering angle)

$$
\frac{z-z_{\mathrm{jet}}}{L} \approx \sqrt{\frac{T}{2 m_{p}} \frac{E_{\mathrm{beam}}+m_{p}}{E_{\mathrm{beam}}-m_{p}}} \approx \kappa \sqrt{A}
$$

Vertically oriented narrow $(3.7 \mathrm{~mm}) \mathrm{Si}$ strips allow us to isolate elastic events as shown by red lines in Fig. 2. Background events are strongly dominated by so called prompts and are expected to have the same time/amplitude distribution for all Si strips.

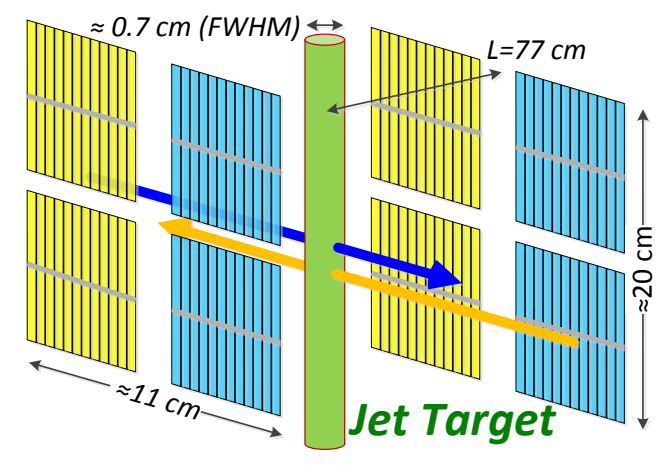

Figure 1: Schematic view of the HJET polarimeter in Run 2015. The detectors, 12 vertical Si strips each, are operationally divided to blue and yellow ones depending on which beam polarization they measure.

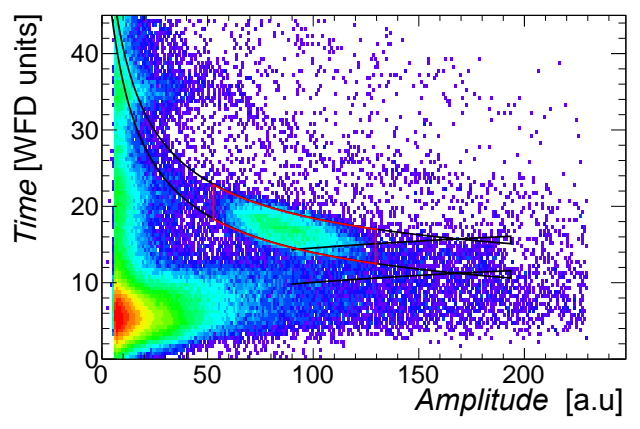

Figure 2: Time/amplitude distributions for detected signals in a Si strip. The WFD time unit equals to $2.369 \mathrm{~ns} .25$ amplitude units approximately corresponds to $1 \mathrm{MeV}$. 


\section{HJET polarimeter upgrade in Run 2015.}

An upgrade of the HJET polarimeter in RHIC Run 15 [4] included:

- new Hamamatsu Photonics K.K. S10938-3627 Si detectors.

The $470 \mu \mathrm{m}$ thick detector consists of 12 strips of $3.7 \times 45 \mathrm{~mm}^{2}$ size. New detectors compared to old ones have thinner and uniform dead-layer. Pairs of the detectors were wire bonded together to form 12-channel (double length strip) readout detectors. The acceptance was significantly increased. However, elastic protons with energy above $7.8 \mathrm{MeV}$ punch through the detector which require a special analysis to reconstruct the kinetic energy.

- new DAQ based on JLab FADC250 [6] waveform digitizers.

The original HJET DAQ (commissioned in 2004) was build using CAMAC 8 bit $140 \mathrm{MHz}$ (effectively $420 \mathrm{MHz}$ ) waveform digitizers [2]. Even though this system still satisfies the main requirements for the HJET DAQ, it was recognized that upgrading to modern WFDs may improve the analysis of systematic errors in polarization measurements.

For the new DAQ we employed FADC250 boards, the 16 channel 12 bit $250 \mathrm{MHz}$ flash ADC designed in Jefferson Lab [6]. The full assembly includes VME64x crate, 6 FADC250 boards, Front Panel Signal Distribution module for the FADC250, and BNL V128 Input/Output register. A Concurrent Technologies VX 915/011-14 Single Board Computer served both as VME controller and the DAQ PC. The FADC250 boards used external $244 \mathrm{MHz}$ Clocks derived from the 28.15 MHz RHIC RF signal. The boards were synchronized by the Bunch Zero signal every jet polarization cycle $(5 \mathrm{~min})$. The V128 polled the NIM levels which manage the jet polarization state.

Relatively low rate in the HJET detectors, $\sim 10 \mathrm{kHz}$ for a $\sim 300 \mathrm{keV}$ threshold, allows us to use the FADC general purpose firmware with a self-trigger capability. Raw waveforms ( 80 samples, about $328 \mathrm{~ns}$ time interval) were recorded and dumped to a network hard drive.

The new VME based DAQ was assembled without destroying the old CAMAC based DAQ. It takes less then 30 minutes to switch (reconnect signal cables) between old and new systems. A software interface to use the new data format with old data analysis was developed which allowed us to migrate to new DAQ smoothly.

\section{Calibration of the HJET polarimeter}

A typical HJET signal waveform is shown in Fig. 3. It was fit using the following parametrization

$$
W(t)=p+A\left(t-t_{i}\right)^{n} \exp \left(-\frac{t-t_{i}}{\tau_{s}}\right)
$$

where $p$ is baseline (pedestal), $A$ is signal amplitude, $t_{i}$ is the proton input time to the detector, and $n$ and $\tau_{s}$ are waveform shape parameters. In data analysis we also used the maximum amplitude time

$$
t_{m}=t_{i}+n \tau_{s}
$$

which is more stable in the waveform fit than $t_{i}$.

The HJET has a capability of exposing of all detectors with two $\alpha$-sources, ${ }^{148} \mathrm{Gd}(3.183 \mathrm{MeV})$ and ${ }^{241} \mathrm{Am}(5.486 \mathrm{MeV}$ ). Such a calibration (Fig. 4) allows us to determine gain $g \sim 2.5 \mathrm{keV} / \mathrm{cnt}$ 


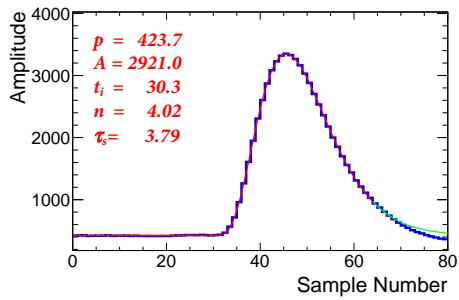

Figure 3: Signal waveform in the HJET. Red line indicate the time interval used for the fit.

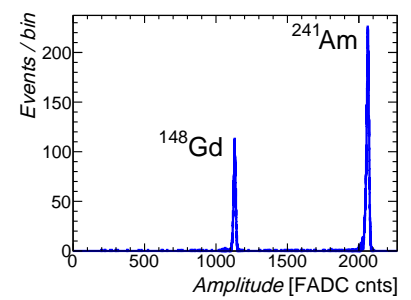

Figure 4: Signal amplitude distribution in the $\alpha$-source calibration.

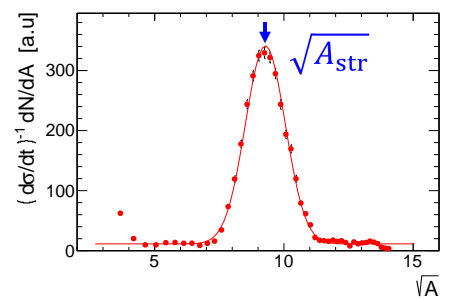

Figure 5: An image of the proton distributions in the jet / beam gas.

and dead-layer thickness $x_{\mathrm{DL}} \sim 0.37 \mathrm{mg} / \mathrm{cm}^{2}$ for every Si strip. The energy resolution found to be $\sigma_{E} \approx 20 \mathrm{keV}$ is dominated by electronic noise. The waveform fit parameters $n^{(\alpha)}$ and $\tau_{s}^{(\alpha)}$ are also determined in this calibration.

Additionally a geometry based calibration was developed [7]. As it follows from Eq. 1.2, for very narrow Si strip the elastic signal amplitude distribution (Fig. 5) may be approximated as

$$
\eta(\sqrt{A})=\left(\frac{d \sigma}{d t}\right)_{\text {elastic }}^{-1} \frac{d N}{d A} \propto f\left(\kappa \sqrt{A_{\mathrm{str}}}-\kappa \sqrt{A}\right)
$$

where $f(z / L)$ is the jet / beam gas hydrogen density along $z$-axis and $\kappa \propto \sqrt{g}$ is a known factor. Finite width of the Si strip results in about $10 \%$ smearing of the distribution width. Contribution of inelastic processes is expected to be a flat background in Fig. 5. Thus, if $z$ coordinate of the strip relative to the jet center is known, the value of $A_{\text {str }}$ found in Gaussian fit of the distribution in Fig. 5 may be related to the kinetic energy $T_{\text {str }} \propto\left(z_{\text {str }}-z_{\text {jet }}\right)^{2}$ calculated using Eq. 1.2. Measurement of recoil proton time $t_{\text {str }}$ corresponding to the $A_{\text {str }}$ allows one to determine $t_{0}$ since the time of flight, $\operatorname{tof}\left(T_{\text {str }}\right)$, is a known function of the proton kinetic energy.

To achieve a satisfactory accuracy of the geometry based calibration, the positions of the Si detectors, as well as corrections due to the bending of recoil proton track in the holding field magnet [2] have to be determined and monitored with accuracy better than $100 \mu \mathrm{m}$. These corrections may be found in a simple way. For given measured energy (amplitude $A_{\mathrm{pr}}$ ), the prompt events time of flight

$$
\Delta t=t_{\mathrm{pr}}\left(A_{\mathrm{pr}}\right)-t_{0}=t_{\mathrm{pr}}\left(A_{\mathrm{pr}}\right)-t_{\mathrm{str}}\left(A_{\mathrm{str}}\right)+\operatorname{tof}\left(T_{\mathrm{str}}\right)
$$

is expected to be the same in all Si strips. Roughly half of all Si strips have a well isolated stopped elastic proton signals. Minimization of the $\Delta t$ distribution for these strips by variation of the corrections to $z$-coordinates solve the problem of geometrical alignment of all Si strips. The distribution of the $\Delta t$ values after alignment is shown in Fig. 6. As a by product, this method proves that time offset $t_{0}$ may be determined with accuracy better than $100 \mathrm{ps}$ using prompt events.

Obviously, $\alpha$-source and geometry based calibrations are uncorrelated. The comparison $\delta_{E}=$ $\frac{T_{\text {str }}-T_{\alpha}}{\left(T_{\text {str }}+T_{\alpha}\right) / 2}$ of the geometrically defined energy $T_{\text {str }}$ with the $\alpha$-calibration calculated energy $T_{\alpha}=$ $g A_{\text {str }}+E_{\text {loss }}\left(g A_{\text {str }}, x_{\mathrm{DL}}\right)$ allows one to estimate systematic errors in the HJET energy calibration (see Fig. 7).

A detailed look on Figs. 6 and 7 indicate some systematic dependence of the $\Delta t$ and $\delta_{E}$ on amplitude $A_{\text {str }}$. This may be explained by disregarded energy loss in dead-layer in the geometry 


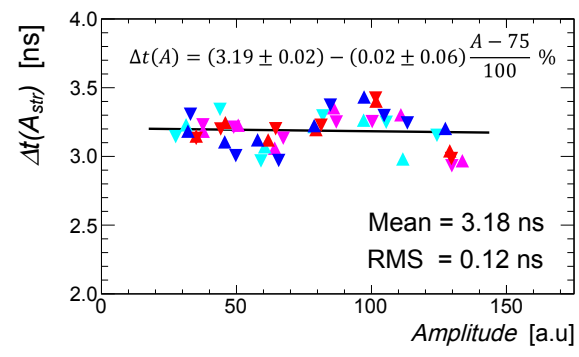

Figure 6: The dependence of the $\Delta t$ on amplitude $A_{\text {str }}$ for $A_{\mathrm{pr}}=15$. Different markers corresponds to different detectors.

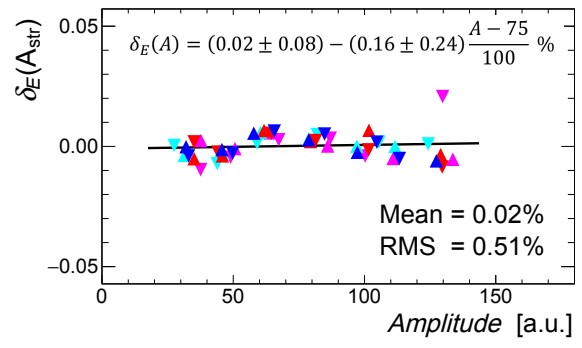

Figure 7: The dependence of the $\delta_{E}$ on amplitude $A_{\text {str }}$. Different markers corresponds to different detectors.

based calibration and by dependence of measured signal time and effective gain $g$ on recoil proton energy (see below). Accounting these corrections promises an essential improvement in the calibration results. However already achieved quality of the calibration is currently sufficient for the needs of polarization measurements in the HJET.

\section{Reconstruction of punched through protons}

The HJET geometrical acceptance in Run 15 allowed us to detect elastic recoil protons with kinetic energy up to $11 \mathrm{MeV}$. Protons with energy more than $7.8 \mathrm{MeV}$ punched through the detector. For such protons the Eq. 1.1 is violated and the larger proton kinetic energy the smaller a signal amplitude is measured. For detected energy above $5 \mathrm{MeV}$ the stopped and punched through protons can not be efficiently separated using only time/amplitude distributions described above.

In the data analysis it was found that waveform shape parameters $n$ and $\tau_{s}$ depend on signal amplitude [8] which may be employed to separate stopped and punched through protons. Since there is a strong correlations between fluctuations of $n$ and $\tau_{s}$ in the waveform fit, it was found convenient to fix the parameter $\tau_{s}=\tau_{s}^{(\alpha)}$ in the fit. To reconstruct a punched through proton kinetic energy one needs the dependence of corrections to measured time $\delta t(T)$, amplitude $\delta A(T)$, and waveform parameter $n(T)$ on proton kinetic energy $T$. In this work the corrections, shown in Fig. 8, were determined by the simulation [8] of charge collection in the Si strips. The simulation parametrization was adjusted using waveform fits in the $\alpha$-calibration. The experimentally measured dependence
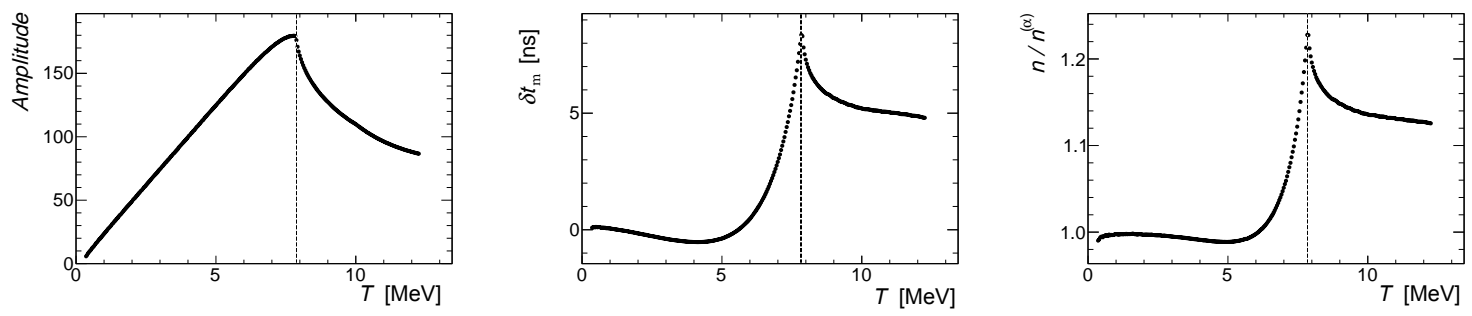

Figure 8: Simulation of waveform shape parameters dependence on recoil proton energy. Vertical dashed lines indicate the threshold energy of $7.8 \mathrm{MeV}$ for punched through protons. 


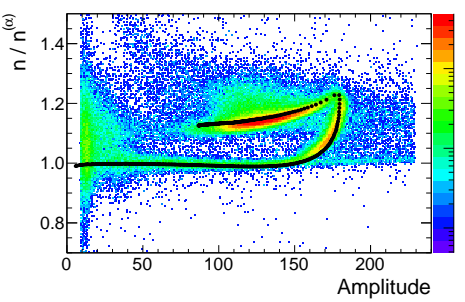

Figure 9: Measured dependence of fit parameter $n$ on signal amplitude. Black line is the simulated dependence.
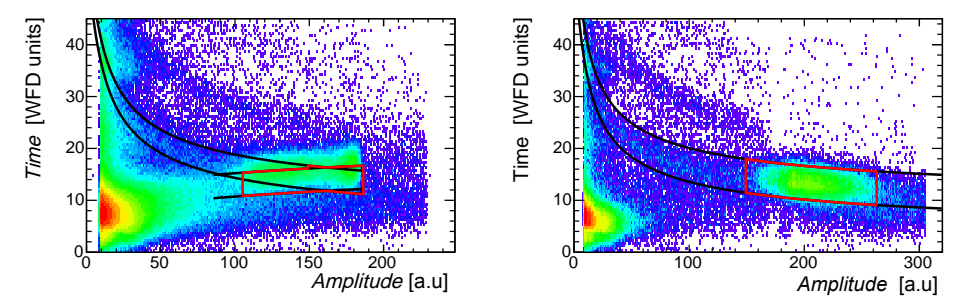

Figure 10: Measured time vs amplitude dependence before (left) and after (right) applying waveform shape cut. Color levels (logarithmic scale) are the same for both plots.

of the fit parameter $n$ on signal amplitude $A$ for all (elastic and background) events in a Si strip is compared with a calibrated (simulated) dependence for elastic protons in Fig. 9. For every point $(n, A)$ consistent with the calibrated dependence, the corrections to measured time and amplitude can be calculated and applied. The time/amplitude distributions before and after (including cuts) correction are shown in Fig. 10. One can see that the corrected time/amplitude dependence for punched through protons matches well the event selection cuts derived from $\alpha$-calibration and prompt time. Also it should be pointed out that the background is strongly suppressed for stopped protons energies.

\section{Systematic errors in absolute polarization measurements}

Determination of the (vertical) beam polarization is based on measurement of left/right asymmetry of recoil proton production in elastic $p p$ scattering. Comparison of asymmetry relative to beam $\left(a_{\text {beam }}\right)$ and jet $\left(a_{\text {jet }}\right)$ polarizations allows one to relate beam polarization $P_{\text {beam }}$ to the jet polarization $P_{\text {jet }} \approx 0.96$ known with a high precision.

$$
P_{\text {beam }}=a_{\text {beam }} / A_{N}, \quad A_{N}=a_{\text {jet }} / P_{\text {jet }}
$$

For elastic $p p$ scattering the analyzing power $A_{N}$ is the same for beam and jet measurements.

Flipping polarities of the proton beams and hydrogen target jet allows us to suppress systematic errors due to the beam intensity and acceptance asymmetries in both, beam and jet, measurements [9]

$$
a=\frac{\sqrt{N_{L}^{\uparrow} N_{R}^{\downarrow}}-\sqrt{N_{R}^{\uparrow} N_{L}^{\downarrow}}}{\sqrt{N_{L}^{\uparrow} N_{R}^{\downarrow}}+\sqrt{N_{R}^{\uparrow} N_{L}^{\downarrow}}}
$$

where $N_{L R}^{\uparrow \downarrow}$ is number of selected events in left/right detectors depending on beam or jet proton polarization.

Since about $r \approx 5 \%$ of events selected for polarization measurements come from beam proton interactions with the beam gas and HJET frame the measured polarization may be biased

$$
P_{\text {meas }}=P_{\text {beam }} \frac{A_{N}+r A_{N}^{(b)}}{A_{N}+r A_{N}^{(j)}}
$$




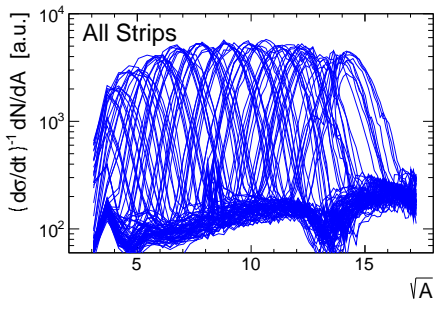

Figure 11: Overlapping of the $\eta(\sqrt{A})$ distributions for all Si strips. A background dependence on $\sqrt{A}$, common for all strips, is clearly seen.
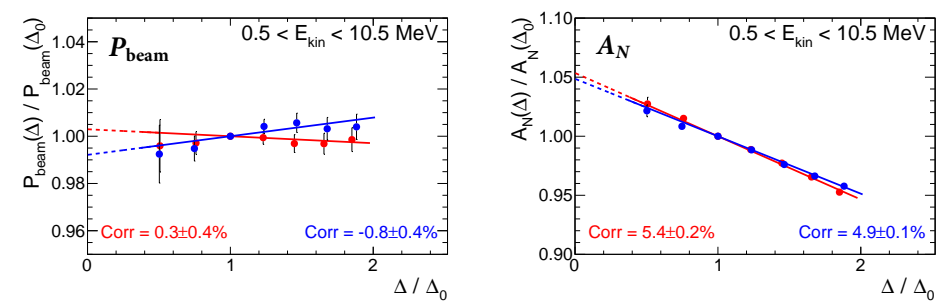

Figure 12: The dependence of measured $P_{\text {beam }}$ and $A_{N}$ on event selection cut $\Delta$ for both RHIC beams. Corrections to the measured values due to background contributions (evaluated at $\Delta \rightarrow 0$ ) are shown on the pictures.

where $A_{N}$ is the analyzing power for elastic $p p$-scattering and $A_{N}^{(b, j)}$ are effective analyzing powers for background events relative to beam and jet polarization, respectively. Generally, polarization measurement is unbiased if $A_{N}^{(j)}=A_{N}^{(b)}$ or r $=0$; For most of the background components one may expect $A_{N}^{(j)}=0$. An important source of systematic errors is a contamination of the jet by hydrogen atoms bound into unpolarized proton molecule. For the molecular hydrogen $A_{N}^{(b)}=A_{N}, A^{(j)}=0$. The molecular hydrogen contamination was experimentally evaluated as $r \sim(3.5 \pm 2.0) \%$ [2].

Since all backgrounds, including molecular hydrogen, are expected to have a "flat" contribution to the $\eta(\sqrt{A})$ distribution, the background events potentially may be counted (see Fig. 11) and subtracted from the data. However this method was not implemented yet.

Event selection includes a $\sqrt{A} \in \sqrt{A_{\text {str }}} \pm \Delta_{0}$ cut with $\Delta_{0}=0.4 \mathrm{MeV}^{1 / 2}$. Extrapolation the dependence of measured beam polarization on the cut parameter $\Delta \rightarrow 0$ allows us to make a background free estimate of the beam polarization (see Fig. 12). Preliminary results are very promising and indicate that systematic errors due to backgrounds (including molecular hydrogen) may be controlled at sub-percent level. However verification of the method is still needed. This verification should include background subtraction described above, comparison with other methods [3], and new direct measurements of molecular hydrogen contamination in the jet.

It was found in the Run 15 data analysis that the HJET negative polarization cavity induces electronic noise (Fig. 13) in two blue detectors (Si strips 37-48 and 73-84) which results in a correlation between event selection efficiency and polarization state of the jet. Such a correlation violates the applicability of the systematic error suppression (Eq. 5.2) in the jet asymmetry $a_{\text {jet }}$ measurement. Thus, for the blue beam, the measured analyzing power and consequently the beam polarization appeared to be corrupted especially for low energy recoil proton energies $T<3 \mathrm{MeV}$. Methods of suppression of this effect at hardware level and in data analysis are being investigated.

\section{Analyzing Power for $\mathbf{p}^{\uparrow} \mathbf{A}$ elastic scattering}

In part of Run 15 the yellow ring was filled with $100 \mathrm{GeV} / \mathrm{n} \mathrm{Au}$ and $\mathrm{Al}$ nuclei [10]. It was found that time/amplitude distributions (Fig. 14) of recoil protons produced by Au/Al scattering on the jet hydrogen atoms is consistent (Fig. 15) with elastic scattering. More detailed analysis shows 


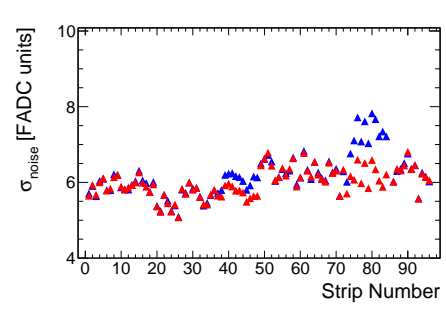

Figure 13: Comparison of electronic noise for all 96 strips. Blue points are for negative jet polarization and red points are for positive.

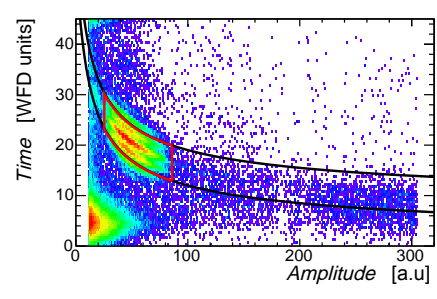

Figure 14: time/amplitude for Gold beam. The time structure for elastic events is attributed to the beam bunch structure.

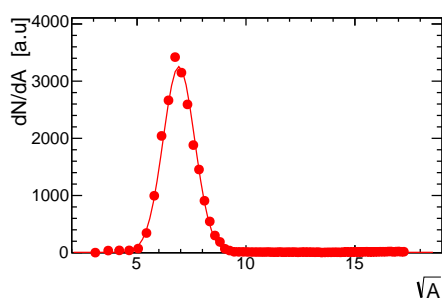

HJET strip Figure 15: The $\eta(\sqrt{A})$ distribudistribution tion for recoil protons from Gold scattering on jet protons.

that contribution of inelastic scattering $A u / A l+p \rightarrow p+X$ does not exceed few percent compared to elastic scattering.

Since scattering of the $100 \mathrm{GeV} / \mathrm{n} \mathrm{Au}(\mathrm{Al})$ beam on polarized protons is equivalent to scattering of $100 \mathrm{GeV}$ polarized proton beam on $\mathrm{Au}(\mathrm{Al})$ nuclei we were able to measure the 100 $\mathrm{GeV}$ proton nucleus $p^{\uparrow} \mathrm{A}$ analyzing power for $\mathrm{Au}$ and $\mathrm{Al}$ as a function of momentum transfer $t=\left(p_{\text {out }}-p_{\text {in }}\right)^{2}=-2 m_{p} T$. The preliminary results are shown in Fig. 16.

Interesting to note that filling of RHIC rings with carbon beam may allow us to measure p-Carbon analyzing power which would be very helpful for understanding the performance of pCarbon polarimeters used in AGS/RHIC [12].

\section{Summary}

New DAQ based on VME 12 bit $250 \mathrm{MHz}$ FADC250 for RHIC HJET polarimeter was assembled, tested, and employed in RHIC Run 2015. Different calibration methods were tested. Energy resolution $\sigma_{E} \sim 20 \mathrm{keV}$ is dominated by electronic noise. Systematic errors in energy calibration is $\delta E / E<0.5 \%$ for $1-6 \mathrm{MeV}$ protons. Time alignment of electronic channels is better than $120 \mathrm{ps}$. $z$-coordinates of detectors may be monitored with accuracy $\delta z \leq 100 \mu \mathrm{m}$. A full reconstruction of punch through protons allowed us to increase recoil proton energy range to $0.5-11 \mathrm{MeV}$ and suppress background for stopped protons. Possible sources of systematic errors in the HJET polar-

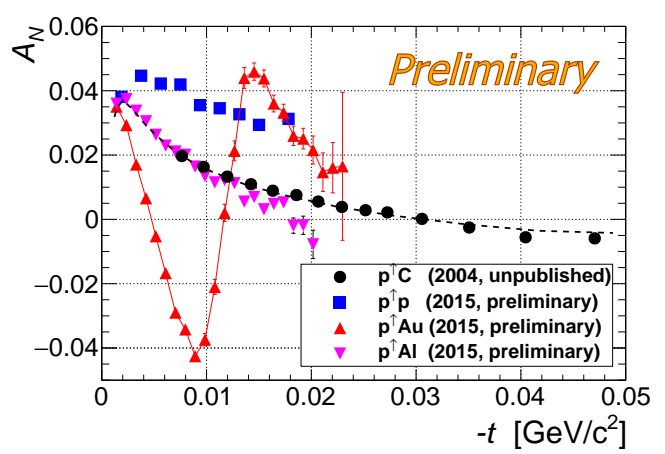

Figure 16: Preliminary results for analyzing power for $100 \mathrm{GeV}$ polarized proton scattering on $p, A u$, and Al measured in Run 15 . Only a small $(\sim 10 \%)$ part of all data was included in the analysis. Background contribution was not subtracted. For comparison, unpublished results [11] obtained at RHIC p-Carbon polarimeter in 2004 are also shown. 
ization measurement were discussed. Preliminary results for measurement of analyzing power of polarized proton scattering on $p, A u$ and $A l$ were presented.

\section{Acknowledgments}

In Run 15 we used FADC250 modules on loan from Jefferson Lab. We acknowledge the outstanding contribution of Chris Cuevas, Hai Dong, Ed Jastrzembski, and Bryan Moffit from JLab Fast Electronics Group to the development the new DAQ for the HJET polarimeter at RHIC.

This work is performed under Brookhaven Science Associates, LLC, contract No. DE-AC0298CH10886 with the U.S. Department of Energy. Funding is also provided from the RIKEN BNL Research Center.

\section{References}

[1] A. Zelenski, Polarized beams in AGS and RHIC in Run-2015, these proceedings POS (PSTP2015) 047

[2] H. Okada et al., Measurement of the analyzing power $A_{N}$ in pp elastic scattering in the CNI region with a polarized atomic hydrogen gas jet target,Phys.Lett. B 638 (2006) 450.

I. G. Alekseev et al., Measurements of single and double spin asymmetry in pp elastic scattering in the CNI region with a polarized atomic hydrogen gas jet target, Phys. Rev. D 79, 094014 (2009).

[3] O. Eyser, Absolute polarimetry of proton beams at RHIC, these proceedings POS (PSTP 2015) 024

[4] V. Schoefer et al., RHIC Polarized proton-proton operation at $100 \mathrm{GeV}$ in Run 15, in Proceedings of IPAC2015, Richmond, VA, USA, paper TUPWI060, pp. 2384-2386.

[5] M.J. Berger et al., (2005), ESTAR, PSTAR, and ASTAR: Computer Programs for Calculating Stopping-Power and Range Tables for Electrons, Protons, and Helium Ions (version 1.2.3), http://physics.nist.gov/Star.

[6] H. Dong et al., Integrated tests of a high speed VXS switch card and 250 MSPS flash ADCs, 2007. doi:10.1109/NSSMIC.2007.4436457.

[7] A. Poblaguev, A precise in situ calibration of the RHIC H-Jet polarimeter, BNL-104-363-2014-IR (March 2014), https://www.bnl.gov/isd/documents/85159.pdf.

[8] A. Poblaguev, Waveform dependence on signal amplitude in the RHIC H-Jet polarimeter, BNL-104-366-2014-IR (February 2014), https://www.bnl.gov/isd/documents/85162.pdf.

[9] G.G. Ohlsen, P.W. Keaton, Techniques for measurement of spin-1/2 and spin-1 polarization analyzing tensors, Nucl. Instr. and Meth 109, 41 (1973).

[10] C. Liu et al., RHIC Operations with asymmetric collisions in 2015, BNL-108367-2015-IR (August 2015), www.rhichome.bnl.gov/RHIC/Runs/RhicRun15pA2.pdf

[11] I. Nakagawa et al., p-carbon polarimetry at RHIC, AIP Conf. Proc. 980, 380 (2008). doi:10.1063/1.2888112

[12] G. Webb, Relative polarization measurements of proton beams using a thin carbon target at RHIC, these proceedings POS (PSTP2015) 035 